\title{
Asymmetric Protonation of Ketone Enolates Using Chiral $\beta$-Hydroxyethers: \\ Acidity-tuned Enantioselectivity
}

\section{Supporting Information}

\author{
B. Moon Kim, ${ }^{*}$ Hyunwoo Kim, Woosung Kim, Keun Young Im and Jin Kyoon Park \\ School of Chemistry \& Molecular Engineering, and Center for Molecular Catalysis, Seoul \\ National University, Seoul, 151-747, Korea
}

\section{Table of Contents}

1. Synthetic scheme, spectral characteristics and elemental analysis data of compounds $\mathbf{1 a}, \mathbf{1} \mathbf{b}$, 1c, 1d, 1e, and 1f.

\section{Pages S1-S3}

2. A representative procedure for the enantioselective protonation of lithium enolates prepared from $4,6,7$, and 8

\section{Page S4}

\section{Experimental}

Compound 1a was prepared according to the literature procedure, ${ }^{13}$ Compounds $\mathbf{1 b}-\mathbf{f}$ were prepared following methods used for the preparation of 1a and their physical data are listed below. 


\section{Synthetic scheme}
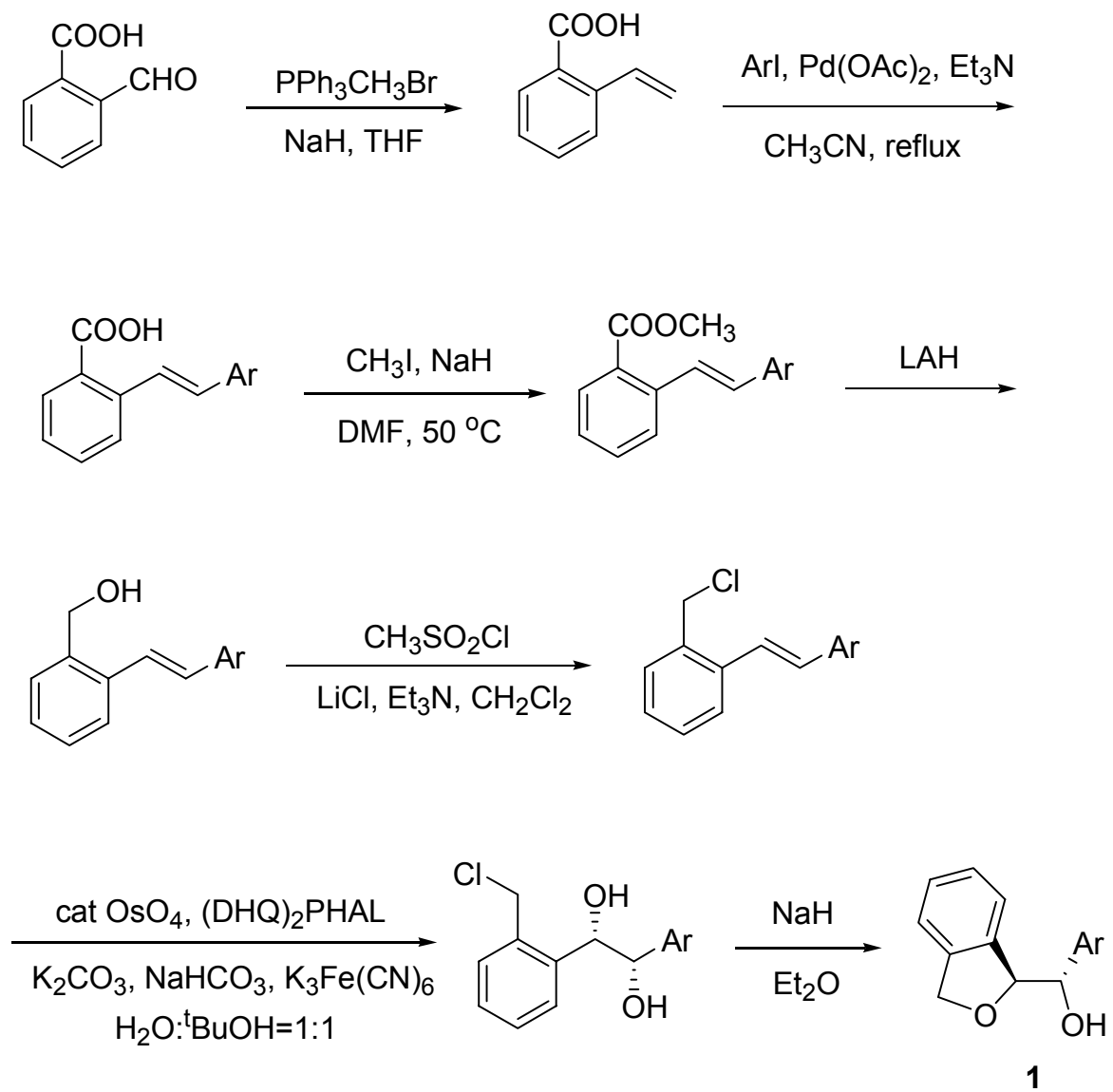

\section{Characterization data}

Compound 1a: IR $\left(\mathrm{KBr}, \mathrm{cm}^{-1}\right) 3430.0,3037.8,2933.0,2868.2,1458.4 ;[\alpha]_{\mathrm{D}}{ }^{25}=+9.3(c 1.0$, $\left.\mathrm{CHCl}_{3}\right) ;{ }^{1} \mathrm{H} \mathrm{NMR}\left(300 \mathrm{MHz}, \mathrm{CDCl}_{3}\right) \delta$ 2.20-3.10 (br, s, $\left.1 \mathrm{H}\right), 4.66(\mathrm{~d}, 1 \mathrm{H}, J=6.8 \mathrm{~Hz}) 5.05$ (d, $1 \mathrm{H}$, $J=12.8 \mathrm{~Hz}) 5.18\left(\mathrm{AB}_{\mathrm{q}}, J=12.8 \mathrm{~Hz}, 2 \mathrm{H}\right), 5.33(\mathrm{~d}, 1 \mathrm{H}, J=6.8 \mathrm{~Hz}), 6.46(\mathrm{~d}, 1 \mathrm{H}, J=7.5 \mathrm{~Hz}) ;{ }^{13} \mathrm{C}$ NMR (75 MHz, $\left.\mathrm{CDCl}_{3}\right) \delta 73.1,77.4,88.5,121.3,123.2,127.4,128.2,128.4,128.8,128.8$, 138.0, 139.6, 140.1; CI Mass m/z $209\left(\mathrm{M}+\mathrm{H}_{-} \mathrm{H}_{2} \mathrm{O}\right)^{+}$

Compound 1b: IR (KBr) 3436.8, 3018.90, 2914.4, 2861.9, 1605.4, 1462.9, 1033.9; $[\alpha]_{\mathrm{D}}{ }^{26}={ }^{-}$ 19.3 (c 1.0, $\left.\mathrm{CHCl}_{3}\right) ;{ }^{1} \mathrm{H}$ NMR (300 MHz, $\left.\mathrm{CDCl}_{3}\right) \delta 2.32$ (s, 6H), 2.83 (br, s, 1H), 4.59 (d, J=6.8 $\mathrm{Hz}, 1 \mathrm{H}), 5.11$ (d, $J=12.3 \mathrm{~Hz}, 1 \mathrm{H}), 5.20(\mathrm{ABq}, J=12.23 \mathrm{~Hz}, 2 \mathrm{H}), 5.32$ (d, $J=6.8 \mathrm{~Hz}, 1 \mathrm{H}), 6.47$ $(\mathrm{d}, J=7.6 \mathrm{~Hz}, 1 \mathrm{H}), 6.98-7.29(\mathrm{~m}, 6 \mathrm{H}) ;{ }^{13} \mathrm{C} \mathrm{NMR}\left(75 \mathrm{MHz}, \mathrm{CDCl}_{3}\right) \delta$ 21.8, 77.3, 88.4, 121.3, 123.2, 125.9, 126.3, 127.3, 128.4, 130.4, 138.2, 138.3, 139.5, 140.1; HRMS (EI) m/z calcd 254.1307 for $\mathrm{C}_{17} \mathrm{H}_{18} \mathrm{O}_{2}[\mathrm{M}]+$, found 254.1316 . 
Compound 1c: IR (KBr) 3347.5, 3070.5, 2880.6, 1589.8, 1568.4, 1426.6, 1198.1, 1041.2; (S,S)1c: $[\alpha]_{\mathrm{D}}^{28}=+25.1\left[c 1.0, \mathrm{CHCl}_{3,}\right] ;(R, R)-\mathbf{1 c}[\alpha]_{\mathrm{D}}{ }^{28}=-21.8\left[c 1.0, \mathrm{CHCl}_{3}\right] ;{ }^{1} \mathrm{H}$ NMR $(300 \mathrm{MHz}$, $\left.\mathrm{CDCl}_{3}\right) \delta 2.88(\mathrm{br}, \mathrm{s}, 1 \mathrm{H}), 4.69(\mathrm{~d}, J=5.7 \mathrm{~Hz}, 1 \mathrm{H}), 5.17(\mathrm{ABq}, J=12.4 \mathrm{~Hz}, 2 \mathrm{H}), 5.29$ (d, J=5.6, $1 \mathrm{H}), 6.75(\mathrm{~d}, J=4.8 \mathrm{~Hz}, 1 \mathrm{H}), 7.11-7.44(\mathrm{~m}, 6 \mathrm{H}) ;{ }^{13} \mathrm{C} \mathrm{NMR}\left(75 \mathrm{MHz}, \mathrm{CDCl}_{3}\right) \delta$ 73.4, 76.0, 87.9, 121.6, 122.7, 126.5, 127.7, 128.6, 128.8, 135.3, 137.3, 140.1, 143.5; EA calcd for $\mathrm{C}_{15} \mathrm{H}_{12} \mathrm{Cl}_{2} \mathrm{O}_{2}$; C,61.04; H,4.10; Cl:24.02; O,10.84. found: C, 60.81; H, 4.06 .

Compound 1d: IR (KBr) 3411.3, 3034.0, 2928.3, 2867.9, 1490.6, 1400.0, 1067.9; $[\alpha]_{\mathrm{D}}{ }^{18}=-12.2$ (c 1.0, $\mathrm{CHCl}_{3}$ ); ${ }^{1} \mathrm{H}$ NMR (300 MHz, $\mathrm{CDCl}_{3}$ ) $\delta 2.95$ (br, s, $\left.1 \mathrm{H}\right), 4.66(\mathrm{~d}, J=5.7 \mathrm{~Hz}, 1 \mathrm{H}), 5.12$ (ABq, $J=12.3 \mathrm{~Hz}, 2 \mathrm{H}), 5.28(\mathrm{~d}, J=5.7 \mathrm{~Hz}, 1 \mathrm{H}), 6.54(\mathrm{~d}, J=7.5 \mathrm{~Hz}, 1 \mathrm{H}), 7.11-7.36(\mathrm{~m}, 6 \mathrm{H}) ;{ }^{13} \mathrm{C}$ NMR $\left(75 \mathrm{MHz}, \mathrm{CDCl}_{3}\right) \delta 73.2,76.6,88.3,121.4,123.0,127.5,128.6,128.9,129.5,134.4$, 137.6, 138.2, 140.1; EA calcd for $\mathrm{C}_{15} \mathrm{H}_{13} \mathrm{Cl}_{1} \mathrm{O}_{2} ; \mathrm{C}, 69.10 ; \mathrm{H}, 5.03 ; \mathrm{Cl}, 13.60 ; \mathrm{O}, 12.27$. found: $\mathrm{C}$, $69.01 ; \mathrm{H}, 5.01$.

Compound 1e: IR (KBr) 3368.1, 2870.7, 1372.3, 1285.1, 1166.2, 1122.0; $[\alpha]_{\mathrm{D}}{ }^{19}=+36.6(c 1.0$, $\left.\mathrm{CHCl}_{3}\right) ;{ }^{1} \mathrm{H}$ NMR $\left(300 \mathrm{MHz}, \mathrm{CDCl}_{3}\right) \delta 2.90(\mathrm{~d}, J=3.5 \mathrm{~Hz}, 1 \mathrm{H}), 4.93(\mathrm{~d}, J=5.0 \mathrm{~Hz}, 1 \mathrm{H}), 5.09$ (s, 2H), $5.35(\mathrm{~d}, J=5.2 \mathrm{~Hz}, 1 \mathrm{H}), 6.80(\mathrm{~d}, J=7.7,1 \mathrm{H}), 7.21-7.34(\mathrm{~m}, 6 \mathrm{H}), 7.83(\mathrm{~s}, 3 \mathrm{H}) ;{ }^{13} \mathrm{C}$ NMR $(75$

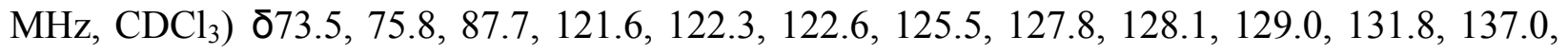
140.1, 142.7; EA calcd for $\mathrm{C}_{17} \mathrm{H}_{12} \mathrm{~F}_{6} \mathrm{O}_{2} ; \mathrm{C}, 56.36 ; \mathrm{H}, 3.34 ; \mathrm{F}, 31.47 ; \mathrm{O}, 8.83$. found: $\mathrm{C}, 56.65 ; \mathrm{H}$, 3.30 .

Compound 1f: IR (KBr) 3418.5, 3078.1, 2865.0, 1619.8, 1325.6, 1164.1, 1123.1, 1067.7; $[\alpha]_{\mathrm{D}}{ }^{19}$ $=+18.3\left(c 1.0, \mathrm{CHCl}_{3}\right) ;{ }^{1} \mathrm{H}$ NMR $\left(300 \mathrm{MHz}, \mathrm{CDCl}_{3}\right) \delta 3.00(\mathrm{~d}, J=3.1 \mathrm{~Hz}, 1 \mathrm{H}), 5.16(\mathrm{ABq}$, $J=12.2 \mathrm{~Hz}, 2 \mathrm{H}), 5.35(\mathrm{~d}, J=5.8 \mathrm{~Hz}, 1 \mathrm{H}), 6.52(\mathrm{~d}, J=7.5 \mathrm{~Hz}, 1 \mathrm{H}), 7.17-7.34(\mathrm{~m}, 3 \mathrm{H}), 7.51(\mathrm{~d}$, $J=8.1 \mathrm{~Hz}, 2 \mathrm{H}), 7.65(\mathrm{~d}, J=8.2 \mathrm{~Hz}, 2 \mathrm{H}) ;{ }^{13} \mathrm{C}$ NMR $\left(75 \mathrm{MHz}, \mathrm{CDCl}_{3}\right) \delta 73.3,76.6,88.1,121.5$, $122.8,125.6,126.3,127.6,128.3,128.7,130.8,137.5,140.1,143.9$; EA calcd for $\mathrm{C}_{16} \mathrm{H}_{13} \mathrm{~F}_{3} \mathrm{O}_{2} ; \mathrm{C}$, 65.30; H, 4.45; F, 19.37; O,10.87. found C, 65.40; H, 4.41. 


\section{A representative procedure for the enantioselective protonation of lithium enolates}

prepared from 4, 6, 7, and 8: TMS enol ether 4 prepared from ketone 5 (23 mg, $0.10 \mathrm{mmol}$ ) in diethyl ether $(1.0 \mathrm{~mL})$ at $0^{\circ} \mathrm{C}$ was treated with a solution of methyl lithium in diethyl ether $(1.40 \mathrm{M}, 110 \mu \mathrm{L}, 0.15 \mathrm{mmol})$. The mixture was stirred at room temperature for $30 \mathrm{~min}$ and then cooled to $-78^{\circ} \mathrm{C}$. Then the lithium enolate solution at $-78^{\circ} \mathrm{C}$ was added to a solution of $\beta$ hydroxyether 1a $(36 \mathrm{mg}, 0.16 \mathrm{mmol})$ in dichloromethane $(1.6 \mathrm{~mL})$ at the same temperature. The mixture was stirred for $1 \mathrm{~h}$ and then gradually warmed up to $0^{\circ} \mathrm{C}$. The reaction mixture was treated with phosphate buffer ( $\mathrm{pH}$ 7.2) and extracted with ethyl acetate. The residue was purified through column chromatography to give 2-methyl-1-tetralone as a pale red oil (16 $\mathrm{mg}, 99 \%$ yield) and 1a (36 mg, 99\% recovery). HPLC analysis to determine the ee of the product ketone was carried out on Hewlett Packard 1100 series HPLC equipped with diode array detector using Chiralcel OD-H column and eluting with hexane/i-PrOH 99:1 (v/v). 